(RESEARCH ARTICLE)

\title{
Anti-angiogenic properties of "Makahiya", Mimosa pudica L. (Fabaceae) aqueous root extracts
}

\author{
Masagca Jimmy T, Publico Krizza May T * and Evangelista Abelisa G \\ Natural Science Department, College of Arts and Sciences Catanduanes State University-Main Campus Calatagan, Virac \\ 4800, Catanduanes.
}

Publication history: Received on 01 August 2019; revised on 18 November 2019; accepted on 21 November 2019

Article DOI: https://doi.org/10.30574/wjarr.2020.5.1.0048

\begin{abstract}
This study was conducted to determine the anti-angiogenic effects of different concentrations of mimosine, flavonoids, tannins, etc. from aqueous root extract (ARE) of Mimosa pudica L. (Fabaceae) on the chorioallantoic membrane (CAM) of developing duck embryo. The extraction method was done by air-drying, grounding and decoction. The AREs of $M$. pudica were prepared at different concentrations (10\%, 20\% and 30\%). These were administered on the CAM of the 10 -day old duck embryos. After $48 \mathrm{~h}$ upon administration of the extracts, the secondary branching points on the CAM are counted and compared with the control groups and with each of the experimental groups. The results reveal that AREs of $M$. pudica inhibited anti-angiogenesis. Results show that $10 \%, 20 \%$ and $30 \%$ concentrations have significantly yielded anti-angiogenic results. There is a significant difference between all the control groups and the experimental groups indicating that as the concentration increases, the branching point of the embryos decreases. This suggests the potential use of the $M$. pudica root extract for further studies on abnormal angiogenesis and evasion of apoptosis as hallmarks in cancer treatment.
\end{abstract}

Keywords: Cancer; Tumor; Aqueous extract; "dormido"; Apoptosis; Novel vasculature

\section{Introduction}

Angiogenesis is the process of developing new blood vessels and controlled by certain chemicals produced in the body [1]. It is critically important during the normal development of the embryo and fetus. The formation of new vasculature from the already existing blood vessels is a characteristic phenomenon in numerous diseases such as tumor formation, rheumatoid arthritis, diabetic retinopathy and psoriasis [2, 3]. During cancer progression, the newly formed tumorassociated blood vessels serve first as nurturing tubes for a growing tumor and next, as conduits for dissemination of tumor cells that escaped from an established primary tumor. Therefore, control of tumor angiogenesis has become a central issue in the fight against cancer progression since anti-cancer therapy could be ineffective once tumor cells reach favored secondary organs and generate metastatic foci.

Abnormal angiogenesis and evasion of apoptosis are the hallmarks of cancer [4]. Accordingly, anti-angiogenic and proapoptotic therapies are effective strategies for cancer treatment. Medicinal plants such as Eugenia jambolana (Myrtaceae), Musa paradisiaca L. (Musaceae) and Coccinia indica Wight \& Arn. (Cucurbitaceae) have been investigated recently for their anti-cancer potential [4]. This indicates wider latitudes for studying other medicinal plants such as the genus Mimosa (Fabaceae), thus this study.

In order to analyze the mechanisms underlying normal and pathological angiogenesis, numerous in vivo angiogenic assays have been established employing different species of laboratory animals, including mammals [mouse, rat, hamster, and rabbit), birds (chicken and quail), and fish (mainly zebra fish) [5] Drug development for the treatment of

\footnotetext{
${ }^{*}$ Corresponding author

E-mail address: KrizzaKMP@yahoo.com
} 
angiogenesis-related diseases has been a continuous pursuit. In vivo and in vitro researches conducted on natural products as potential source of novel drugs to treat several human diseases indicated promising results [4, 6, 7, 8]. Among the in vivo models, the chick embryo chorioallantoic membrane (CAM) has been used to implant several tumor types as well as malignant cell lines to study their growth rate, angiogenic potential and metastatic capability [9].

\subsection{Studies on M. pudica}

Recent studies include that on adverse effects of tannin contained in $M$. pudica L root extract [10]; on assisted green synthesis and photoluminescence studies [11]; on use flower extract in the synthesis of highly stable gold nanoparticles (AuNPs) [12]; and wound healing potential of the ethanol extract of the whole M. pudica L. [13]. Karthikeyan \& Chandran [14] investigated the pharmacognostic and phytochemical aspects on the extractive values for detecting adulteration; while the study of Mahadevan et al. [15] demonstrated the neuroprotective effects of M. pudica L. on against MPTP induced Parkinsonism in mice model.

Previous works on the use of the root part of the plant as the angiogenesis inhibitor was based on the phytochemical tests indicating the presence of phytochemical compounds [16] and the study of Ranjan, Kumar, Seethalakshmi, \& Rao [17]. It was proposed that several CAM angiogenic assays have been introduced since almost a century ago when rat Jensen sarcoma cells, implanted into the CAM on Day 6 of incubation, were demonstrated to develop large tumors showing signs of tumor-induced angiogenesis. The chick embryo's chorioallantois is formed between days 4 and 5 of development, when the outer mesodermal layer of the allantois fuses with the mesodermal lining of the chorion, and a network of blood vessels is gradually formed between the two layers. The central portion of the CAM is fully developed by Day 8 to 10 at which time it becomes capable of sustaining tissue grafts, while the outskirts of the CAM are still developing and expanding until the CAM fully envelopes the embryo at Day 12 of incubation. Histologically, the CAM consists of three germ layers, that is, ectoderm, mesoderm, and endoderm. M. pudica L. contains mimosine, which is a toxic alkaloid. Adrenalin like substance has been identified in the extract of its leaves. M. pudica L. is known to possess sedative, emetic and tonic properties, and has been used traditionally in the treatment of various ailments including alopecia, diarrhea, dysentery, insomnia, tumor, and variou urogenital infections [18].

In 1971, Folkman proposed a hypothesis that tumor growth is angiogenesis- dependent. In that report, Folkman showed preliminary evidence that tumors could not enlarge beyond $\mathrm{mm}$ diameters without recruiting new capillary blood vessels (micro vessels). Such hypothesis stated that tumors secreted a diffusible substance that could stimulate end othelial cell proliferation in host capillary blood vessels. Folkman also introduced the concept of "anti-angiogenesis" as a potential novel anticancer therapy. Folkman followed his 1971 hypothesis with supporting evidence indicating that tumors secreted diffusible end othelial mitogens in vivo, which could induce angiogenesis in neighbouring micro vessels. He and his colleagues then showed that tumor growth could be inhibited by blocking angiogenesis, and they proved that diffusible angiogenesis inhibitory factors existed [19].

The weed plant "dormido" (in Catanduanes dialect), M. pudica L. is a small or middle-sized tree, about $1.5 \mathrm{~m}$ tall cultivated throughout India and are found in ubiquitous areas of the island province of Catanduanes as weed population [20]. All parts of the tree are considered to possess medicinal properties and used in the treatment of wounds [13] bleeding, piles, diarrhea [11] biliousness, leprosy, dysentery, vaginal and uterine complaints [21], inflammations, burning sensation, fatigue, asthma, leukoderma, blood diseases [18].

Therefore, present study has been conducted to find out the anti-angiogenic potentials of the aquaeus root extracts (ARE) of "dormido", M. pudica L. (Fabaceae) on the chorioallantoic membrane of developing duck embryo. Further, the study determined the anti-angiogenic properties of $M$. pudica L. aqueous root extract on the vascularization and proliferation of embryonic blood vessels using 10 - day old duck embryos. Specifically, the study described the different gross morphological characteristics on the CAM of 10-day old duck embryos treated with ARE of $M$. pudica L. in terms of its blood vessel proliferation. Significant differences in the effects of concentrations of ARE of M. pudica L. in the assays used and concentrations of ARE of M. pudica L. used in the CAM of 10-day old duck embryo.

\section{Material and methods}

\subsection{Design of the study}

A Complete Block Design is used to assess the potential angiogenesis inhibiting property of the selected plant using the Chorioallantoic Membrane (CAM) Assay. The medicinal plant used in the study M. pudica L. The experiment used three treatments and three replicates having three fertilized duck eggs per treatments that was analysed [22]. 


\subsection{Collection and preparation of plant sample}

Fresh M. pudica L. were collected from Pangilao and Katipunan, San Miguel Catanduanes. Mature root samples were obtained from the plant and washed with tap running water. The root portion of the plant was brushed and cleaned to remove the mud and soil. The roots, specifically the main root of the plant was then cut off from the main plant itself. It was then washed again for 3 times. Distilled water is used in cleaning the roots. The roots were then placed on a tray and air-dried for approximately 3 weeks and/or dried in the oven with at $46^{\circ} \mathrm{C}$. Following Balick \& Weil [23], with the use of a sterile blender, roots were grounded into coarse powder and placed in an air-tight container.

\subsection{Preparation of root extract}

The decoction method of extraction was used in this study. A total of $20 \mathrm{~g}$ of coarse powder of dried roots of M. pudica L. was then decocted in $80 \mathrm{ml}$ of water (1:4 ratio) and boiled until reduced to one-fourth $(20 \mathrm{ml})$ of the total quantity. This process was repeated until $100 \mathrm{ml}$ of the extract is obtained [24]. The extracts of M. pudica L. roots were filtered using Whatman No. 1 filter paper and stored in sterile, air tight containers. These extracts were then diluted with distilled water into different concentrations. The treatment group will be as follows:

Positive Control: $\quad 0.2 \%$ Ethanol Solution

Negative Control: $\quad 0.9 \%$ Normal Saline Solution

Concentrations: $\quad$ 10\% ARE of $M . p$ pudica

$20 \%$ ARE of M. pudica

30\% ARE of M. pudica

\subsection{Collection and incubation of $A$. platyrhynchos eggs}

15 fertilized 8-Day old A. platyrhynchos eggs were obtained from an egg vendor in Constantino, Virac, Catanduanes obtained from a local hatchery in the town of Polangui, Albay. Adopting Maniago et al. [25], the eggs were cleaned with $70 \%$ Ethyl alcohol and placed in an incubator at a constant temperature of $37^{\circ} \mathrm{C}$ for 2 days and extended until on the 10th day prior to the experiment.

\subsection{Egg Candling}

Egg candling was done on the 9th day of incubation with an improvised egg chamber. This method was performed in order to determine if the eggs were fertilized. An egg is confirmed fertilized if a tiny speck on the yolk called the germ spot is present during egg candling. This step is also necessary to ensure that the windowing be performed exactly at the top of the embryo.

\subsection{Chorioallantoic membrane assay}

The test specimens were divided into five treatment groups: one group for the negative control, one group for the positive control and three groups for the crude aqueous root [26]. The incubated eggs were disinfected with $70 \%$ ethyl alcohol. Using a modified egg windowing technique, a $1 \mathrm{x} 1 \mathrm{~cm}$ hole is made to expose the CAM to direct access for experimental manipulation. Using sterile $1 \mathrm{cc}$ syringe, $0.2 \mathrm{ml}$ of the concentrations was administered on each of the experimental specimens. The inoculated site of the treated eggs was sealed with micropore tape and incubated at $37^{\circ} \mathrm{C}$ for 2 (two) days. Day 10 is the subject for experimental treatment because between Day 8 and Day 10, the developing CAM vasculature is ready to sprout in response to additional proangiogenic stimuli, and in turn, is very responsive to angiogenic factors.

\subsection{Evaluation of blood vessel formation of the CAM}

After 48 days of administration, each of the test eggs was removed from the incubator and the vascularization of the CAM was examined and determined. The CAM is harvested by removing the shell of the egg and extracting it from the embryo. The CAMs were photographed using a high-definition camera. The number of small blood vessels are recorded by counting the number of branch points per blood vessel. The number of branch points per blood vessel are then compared with the other concentrations $[1,27,28]$. 


\subsection{Statistical Analysis}

The data gathered are then subjected under One-way ANOVA to determine the significant differences between the treatments. Significant differences among the treatments and concentrations were determined through pair-wise comparison. All statistical analyses were done at $5 \%$ probability level [25].

\section{Results and discussion}

\subsection{Gross morphological observation of CAM}

A total of 15 eggs, 3 eggs per treatment, was used in the study. The aqueous root extract of M. pudica L. extracted via decoction were administered to each of the test eggs on its $10^{\text {th }}$ day of incubation period. Two days after the administration of the aqueous extract, collaterals or branch points were photographed using a high definition camera. Each of the CAMs blood vessel density were counted and tabulated to determine the angiogenic effects of the different concentrations.

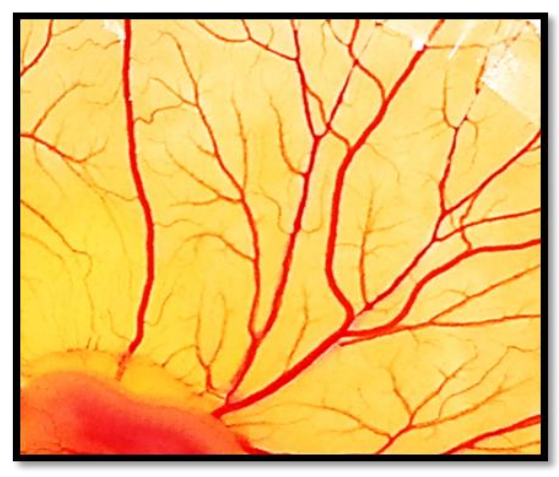

Figure 1 Counting number of branches in the blood vessels from a primary blood vessel (black arrow), vessels that branch from it, the secondary blood vessels (white arrow).

The anti-angiogenic property was apparent for the duck embryos treated with various concentration if $M$. pudica aqueous extract with the embryos treated with highest dose showing the least developed blood vessels which is the $30 \%$ concentration. This ability of the extract to inhibit blood vessel formation shows significance in cancer therapy. Disruption of tumor vasculature through anti-angiogenic treatments would provide a means to starve tumors of their blood supply and nutrient supply which eventually inhibits the spread of cancerous growth [29, 30].

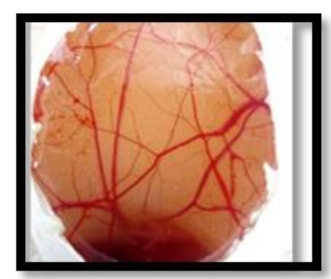

A

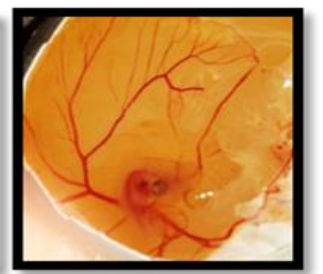

B
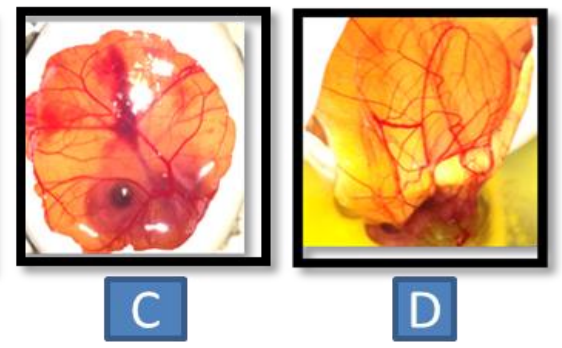

D

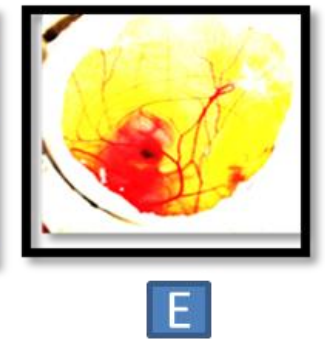

Figure 2 Representative photographs on the effects of root extracts of M. pudica L. on CAM of 10-day old duck embryo treated with $0.9 \%$ saline solution (A); positive control (B); $10 \%$ aqueous $M$. pudica L. aqueous root extract (C); $20 \% M$. pudica L. aqueous extract (D); and with 30\% M. pudica L. aqueous root extract (E).

\subsection{Effects of M. pudica L. aqueous extracts on the angiogenesis of CAM of 10-day old duck embryo}

A total of 15 test eggs, 3 eggs per treatment were used in the study. The ARE of $M$. pudica L. was administered to each test of the test eggs on the 10th day of incubation period. Two days after the administration of crude extracts, secondary collaterals or branch points from the treated areas of CAM were counted and recorded to determine its anti-angiogenic effects. Results of study revealed that AREs of $M$. pudica L. caused reduction in the formation and growth of blood vessels showed that the aqueous extracts of M. pudica L. caused a decrease in the formation of growth of blood vessels (see Table 1). 
Test embryos treated with control groups treated with $0.9 \%$ saline solution and $2 \%$ ethanol formed an average branch point of 121.3 and 22.7. The concentration of $10 \% \mathrm{M}$. pudica L. aqueous extract $\left(\mathrm{T}_{1}\right)$ formed an average of $46.3 \mathrm{branch}$ points while those treated with $20 \%\left(\mathrm{~T}_{2}\right)$ and $30 \%\left(\mathrm{~T}_{3}\right)$ of the aqueous extract formed an average of 35.3 and $27.7 \mathrm{branch}$ points, respectively. This indicates that as the concentration increases, the number of branch points of blood vessel decreases.

Table 1 Average number of branch points from each treatment

\begin{tabular}{ll}
\hline Treatment & Mean of Branch Point Numbers \\
\hline Negative Control & 121.3 \\
Positive control & 22.7 \\
$\mathrm{~T}_{1}$ (10\% ARE of $M$. pudica) & 46.3 \\
$\mathrm{~T}_{2}$ (20\% ARE of M. pudica) & 35.3 \\
$\mathrm{~T}_{3}$ (30\% ARE of M. pudica & 27.7 \\
\hline
\end{tabular}

\subsection{Significant differences in the anti-angiogenic effects of different concentrations of ARE of M. pudica on the CAM of a 10-day old duck embryo}

The decrease in the angiogenesis on the CAM of the test embryos in different experimental treatments clearly indicates that the aqueous extract of $M$. pudica L. was an angiogenesis inhibitor. As the concentration of the aqueous extract increases, the number of branch points formed decreases. Results showed that all comparisons showed significant mean differences. The varying concentrations $(10 \%, 20 \%$, and $30 \%)$ with the negative and positive groups are effective angiogenesis inhibitors. It revealed that the observed F-value (16.98) is greater than the critical value (3.29) at 0.05 level of significance. Hence, there is a significant difference on the angiogenesis of the CAM of the different experimental treatments. The null hypothesis is therefore rejected.

Angiogenesis is an important process in the body both during normal and pathological conditions. The growth and spread of cancer cells especially require the process of formation of blood vessels. Scientific endeavors focused on angiogenesis in tumors resulted in the discovery of new venues for the treatment of cancer patients [4, 29]. Angiogenesis is the growth of neo-vessels from existing vasculature. Usually, angiogenesis is tightly controlled by a balance of angiogenesis factors and inhibitors, and occurs only in embryonic development, and wound healing. To date, the stimulation of angiogenesis using angiogenesis peptides has produced encouraging clinical results in treating coronary artery diseases. Blocking angiogenesis with antibodies of angiogenesis factors or with enzyme inhibitors is effective for treating malignancy but there is room for improvement. Further relevance is the fact that some of the plant-derived anticancer drugs are plant derived [31].

In the present study, the anti-angiogenic potential of M. pudica L. aqueous extract (MPAE) was tested by introducing different concentrations $(10 \%, 20 \%$, and $30 \%)$ using CAM assay. The anti-angiogenic effect on the CAM is quantified by the number of branch points in the blood vessels. Blood vessels were categorized as primary, secondary and tertiary. The primary blood vessels were designated to the thickest blood vessels; secondary blood vessels are the blood vessels branching from the primary blood vessels while tertiary blood vessels are the vessels sprouting from the secondary blood vessels. Branch points or collaterals were counted manually [27, 1]. The effect of ARE of $M$. pudica L. exhibited anti-angiogenic property due to the significant reduction on the number of branch points. There is significant difference in the different concentrations used in the test embryos.

\section{Conclusion}

General morphological analysis of the CAM of the embryos on each experimental group revealed that there is a varying difference between the blood vessels proliferation of the different experimental groups. The varying concentrations (10\%, 20\% and 30\%) of ARE of M. pudica L. showed reduction in both blood vessel formation and branching complexity. There is significance difference between the concentrations, thus, the null hypothesis was rejected. In order to confirm further and accept convincing claim on the effect of ARE of $M$. pudica L. extraction on the extraembryonic blood vessel of duck embryos, it is recommended that similar study will be conducted using different solvent extraction such as methanol, ethanol, hexane, etc. A toxicity study may be conducted to establish the lethal dose of the experimental substance. Finally, the potential use of the ARE of M. pudica L. for further studies on abnormal angiogenesis and evasion of apoptosis as hallmarks in cancer treatment is clearly recognized for future inquiries. 


\section{Compliance with ethical standards}

\section{Acknowledgment}

The first author (KP) expresses her thanks to Dr. Meda B. Mercado for assistance on the statistical analysis.

\section{Disclosure of conflict of interest}

The authors declare no conflicts of interest.

\section{References}

[1] Biscocho HEH and Ching JA. (2016). Effects of crude extracts of various pigments isolated from Spirulina platensis and Chlorella vulgaris on the angiogenesis of chick embrouo chorioallantoic membrane (CAM). Journal of Experimental Biology and Agricultural Sciences, 4(2), 121-126.

[2] Yu ST, Chen TM, Chern JW, Tseng SY and Chen YH. (2009). Down regulation of GSTpi expression by tryptanthrin contributing to sensitization of doxorubicin- resistant MCF-7 cells through c-jun NH2-terminal kinase-mediated apoptosis. Anticancer Drugs, 20, 382-388.

[3] Miao S, Shi X, Zhang H, Wang S, Sun J, Hua W, Miao Q, Zhao Y and Zhang C. (2011). Proliferation-attenuating and apoptosis-inducing effects of tryptanthrin on human chronic myeloid leukemia K562 cell line in vitro. International Journal of Molecular Science, 12, 3831-3845.

[4] Raj MH, Ghosh D, Banerjee R and Salimath BP. (2017) Suppression of VEGF-induced angiogenesis and tumor growth by Eugenia jambolana, Musa paradisiaca, and Coccinia indica extracts, Pharmaceutical Biology, 55(1), 1489-1499.

[5] Romanoff AL. (1960). The avian embryo. Structural and functional development. The avian embryo. Structural and Functional Development.

[6] Cragg GM and Newman DJ. (2013) Natural products: A continuing source of novel drug leads. BiochimBiophysActa, 1830(6), 3670-3695.

[7] Grant RM, Lama JR, Anderson PL, McMahan V, Liu AY, Vargas, L, Goicochea P, Casapía M, Guanira-Carranza JV, Ramirez-Cardich ME and Montoya-Herrera O. (2010). Preexposure chemoprophylaxis for HIV prevention in men who have sex with men. New England Journal of Medicine, 363(27), 2587-2599.

[8] Fotsis T, Pepper M, Adlercreutz H, Hase T, Montesano R and Schweigerer L. (1995). Genistein, a dietary ingested isoflavonoid, inhibits cell proliferation and in vitro angiogenesis. The Journal of nutrition, 125(suppl_3), 790S$797 \mathrm{~S}$.

[9] Ribatti D. (2008). The chick embryo chorioallantoic membrane in the study of tumourangiogenesis. Romanian Journal of Morphology and Embryology, 49(2), 131-135.

[10] Vejayan P. (2016). Adverse effects of tannin contained in Mimosa pudica root extract. Journal of Applied Sciences, 16(10), 477-483.

[11] Venkatachalaiah KN, Venkataravanappa M, Nagabhushana H and Basavaraj RB. (2016). Mimosa pudica (L.) Assisted green synthesis and photoluminescence studies of Y203: Mg2+ nanophosphor for display applications. In IOP Conference Series: Materials Science and Engineering, 149(1), 012177.

[12] Mapala K and Pattabi M. (2017). Mimosa pudica flower extract mediated green synthesis of gold nanoparticles. NanoWorld J, 3(2), 44-50.

[13] Jagetia GC, Lyngdoh R, Lalramchuana and Borah BK. (2017). Mimosa pudica (Lajwanti) accelerates repair and regeneration of deep dermal excision wound in swiss albino mice. International Journal Complement Alternative Medicine, 9(2), 00293.

[14] Ramesh S, Karthikeyan K and Chandran C. (2017). Photochemical screening and pharmacognostic studies on Mimosa pudica L (Sensitive plant). International Journal of Fauna and Biological Studies, 4(4), 170-175.

[15] Mahadevan MV, Ramaswamy RS and Banumathi V. (2017). Mimosa pudica up-regulates Dat and Th proteins xpression, in turn, locomotor functions in mouse Mptp-1-Methyl-4-Phenyl-1,2,3,6-Tetrahydropyridine model of parkinsonism. International Journal of Pharma and BioSciences, 8(1)(B), 24-29. 
[16] Pande M and Pathak A. (2010). Preliminary pharmacognostic evaluations and phytochemical studies on roots of Mimosa pudica (Lajvanti). Int J Pharm Sci Rev Res, 1(1), 50-52.

[17] Ranjan RK, Rao MRK, Sathish Kumar M, and Seethalakshmi I. (2013). Phytochemical analysis of leaves and roots of Mimosa pudica collected from Kalingavaram, Tamil Nadu. Journal of Chemical and Pharmaceutical Research,, 5(5), 53-55.

[18] Joseph B, Georg J and Mohan J. (2013). Pharmacology and Traditional Uses of Mimosa pudica. International Journal of Pharmaceutical Sciences and Drug Research, 5(2), 41-44.

[19] Cao Y and Langer R. (2008). A review of Judah Folkman's remarkable achievements in biomedicine. PNAS.

[20] Masagca JT. (2017). On-going study. Assessing the aphrodisiac activity of xtracts from leaves, stems and roots of the minor mangrove plant, Terminalia catappa ('Tarisoy') and Mimosa pudica ('Dormido') the coastal areas of Catanduanes, Luzon: study I- physical methods (mating behavior). Unpublished Report. Catanduanes State University.

[21] Daar AS, Singer PA, Persad DL, Pramming SK, Matthews DR, Beaglehole R, Bernstein A, Borysiewicz LK, Colagiur S., Ganguly N and Glass RI. (2007). Grand challenges in chronic non-communicable diseases. Nature, 450(7169), 494.

[22] Salas GM and Totaan EV. (2015). Selected Philippine herbal plant extracts as angiogenesis inhibitors using Chick Chorioallantoic Membrane (CAM) assay. International Research Journal of Biological Sciences, 28-32.

[23] Balick MJ and Weil A. (2014). Rodale's 21st-century herbal: a practical guide for healthy living using nature's most powerful plants.

[24] Acharya R, Bhuvad SB, Nariya MB and Nishteswar K. (2014). Comparative anti-inflammatory and analgesic activities of leaf powder and decoction of Chirabilva [Holoptelea integrifolia (Roxb.) Planch]. An International Quarterly Journal of Research in Ayurveda, 35(3), 339-343.

[25] Maniago KGN, Mari CGS and Pareja MC. (2014). Angiogenic effect of Curcuma longa Linn. (Turmeric) tea powder on the chorioallantoic membrane of 10 - day old Annasluzonica (Duck) eggs. Annals of Biological Research, 5 (4), 32-37.

[26] Camposano JE, Dela Torre GLT, Larcia LLH and Laxamana JG. (2016). Screening for the anti-angiogenic activity of selected Philippine medical plants using chorioallantoic membrane assay. Mahidol University Journal of Pharmaceutical Science, 43(4), 173-182.

[27] Olarte EI. (2007). Potential anti-angiogenic property of Cassia alata leaxane extract on the embryonic blood vessels of duck (Annas domesticus) embryos, 1(3), 1-13.

[28] Lontoc KLC. (2012). Angiogenic effect of Talisai (Terminalia catappa L.) crude extract on chorioallantoic membrane of chick embryo. Steth Vol, 6, 42-52.

[29] Theoharides TC, K Singh L, Boucher W, Pang, X, Letourneau R, Webster E and Chrousos G. (1998). Corticotropinreleasing hormone induces skin mast cell degranulation and increased vascular permeability, a possible explanation for its proinflammatory effects. Endocrinology, 139(1), 403-413.

[30] Ghavamzadeh ARDESHIR, Moussavi A, Jahani M, Rastegarpanah M and Iravani M. (2001, April). Esophageal cancer in Iran. In Seminars in oncology, 28(2), 153-157. WB Saunders.

[31] Fan TP, Leung KW, Yeh JC, Yue PYK and Wong RNS. (2006). Angiogenesis: from plants to blood vessels. Trends in Pharmacological Science, 27(6).

\section{How to cite this article}

Masagca JT, Publico KMT and Evangelista AG. (2020). Anti-angiogenic properties of "Makahiya”, Mimosa pudica L. (Fabaceae) aqueous root extracts. World Journal of Advanced Research and Reviews, 5(1), 105-111. 